\title{
INFLUÊNCIA DO TOLTRAZURIL 5\% SOBRE A EXCREÇÃO DE OOCISTOS E GANHO DE PESO EM BEZERRAS LEITEIRAS COM COCCIDIOSE SUBCLÍNICA
}

\author{
ANTUNES, Marcelo Moreira ${ }^{1}$; \\ PEREIRA, Rubens Alves ${ }^{2}$; \\ CASTRO, Natália Ávila de ${ }^{3}$; \\ SCHWEGLER, Elizabeth ${ }^{4}$; \\ RABASSA, Viviane Rohrig \\ RAIMONDO, Raquel Fraga e Silva; \\ FARIAS, Lucas Balinhas ${ }^{7}$; \\ CORRÊA, Marcio Nunes ${ }^{8}$.
}

\footnotetext{
${ }^{1}$ Mestrando em Biotecnologia - UFPEL, ${ }^{2}$ Pós-Doutorando em Veterinária - UFPEL, ${ }^{3}$ Mestranda em Desenvolvimento Regional e Meio Ambiente - Unir, ${ }^{4}$ Professora substituta do IFSC - Campus Araquari, ${ }^{5}$ Professora Ajunta de Semiologia - UFPEL, ${ }^{6}$ Pós-Doutoranda em Veterinária - UFPEL, ${ }^{7}$ Graduando em Medicina Veterinária - UFPEL, ${ }^{8}$ Professor Adjunto de Clínica Médica de Ruminantes - UFPEL / Núcleo de Pesquisa, Ensino e
} Extensão em Pecuária.

\section{RESUMO}

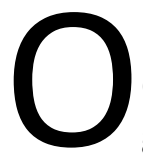

objetivo deste estudo foi demonstrar a eficácia do Toltrazuril 5\% no tratamento da coccidiose subclínica, através da avaliação da excreção de oocistos nas fezes e do ganho de peso de bezerras leiteiras naturalmente infectadas por Eimeria spp. durante as três primeiras semanas de vida. Foram selecionadas 22 bezerras, com 20 e 35 dias de idade, mantidas nas mesmas condições de criação e com presença confirmada de oocistos nas fezes (dia -7 do estudo). As bezerras foram divididas aleatoriamente nos seguintes grupos experimentais: Tratamento $(n=13)$, que receberam uma dose oral de 3 $\mathrm{mL} / 10 \mathrm{~kg}$ de peso vivo $(15 \mathrm{mg} / \mathrm{kg}$ ) de Toltrazuril $5 \%$ no dia 0 , uma semana após a confirmação da eliminação de oocistos (dia -7); e grupo Controle $(n=9)$, que receberam uma dose oral de $3 \mathrm{~mL} / 10 \mathrm{~kg}$ de peso vivo de solução de $\mathrm{NaCl}$ 0,9\%, como placebo. As coletas de fezes para avaliação da eliminação de oocistos foram realizadas nos dias $-7,0$ e 14, enquanto que a avaliação do peso corporal foi realizada nos dias 0,14 e 32 . O tratamento com Toltrazuril $5 \%$ reduziu a eliminação de oocistos e o percentual de bezerras infectadas, além de ter proporcionado um maior peso final para as que receberam o tratamento. Dessa forma, o Toltrazuril 5\% se apresenta como uma alternativa viável para minimizar os efeitos deletérios da coccidiose subclínica e evitar o aparecimento da forma clínica da doença, evitando assim o retardo no desenvolvimento durante a fase inicial de criação.

Palavras-chave: Coccidiose. Toltrazuril. Bezerras leiteiras. Oocistos. GMD. 


\section{INTRODUÇÃO}

Em ruminantes, especialmente em bovinos leiteiros, um adequado desenvolvimento durante as primeiras semanas de vida é importante para um bom desempenho durante sua vida produtiva. Diante disso, estratégias economicamente viáveis que previnam doenças comuns e proporcionem um ganho de peso adequado durante esta fase são fundamentais para a eficiência do sistema de produção (SPADETTO; TAVELA, 2013).

A coccidiose é uma doença cosmopolita causada por protozoários coccídeos do gênero Eimeria spp., que acomete ruminantes criados sob diversas formas de manejo (LIMA, 2004). Bezerras severamente afetadas apresentam sinais clínicos gastrintestinais como diarreia sanguinolenta profusa, além de desidratação, anorexia, fadiga e letargia, podendo resultar em altas taxas de mortalidade (DAUGSCHIES; NAJDROWSKI, 2005; LIMA, 2004).

As perdas econômicas devido à coccidiose são bem conhecidas há algum tempo e podem ser classificadas em perdas pela doença clínica (diarreia e perda de peso) ou infecções subclínicas (baixo ganho de peso) (CHARTIER; PARAUD, 2012). De acordo com Lassen e Ostergaard (2012), os custos associados com a coccidiose chegam à \$2,60 por bezerra/ano. Já Matjila e Penzhorn (2002) estimaram perdas mundiais de rentabilidade na ordem de 400 milhões de dólares ao ano.

A maioria das pesquisas sobre coccidiose tem se direcionado às bezerras e novilhas com elevados níveis de infecção por Eimeria spp. (coccidiose clínica), e suas relações com o ganho de peso durante a fase de desenvolvimento (BANGOURA; DAUGSCHIES, 2007; HOOSHMAND-RAD et al., 1994; MUNDT et al., 2005a). Entretanto, apenas 5\% dos animais infectados apresentam sinais clínicos de coccidiose (MUIRHEAD, 1989) o que torna as infecções subclínicas tão ou mais importantes que as clínicas, pois são mais prevalentes e também reduzem a ingestão de alimentos, diminuem a eficiência alimentar, prejudicando o desenvolvimento dos animais (MATJILA; PENZHORN, 2002).

Em geral, o tratamento da coccidiose clínica é considerado ineficaz em reestabelecer a funcionalidade completa das vilosidades intestinais afetadas, ou seja, os animais que se recuperam de infecções severas geralmente sofrem perdas produtivas permanentes (ERNST; BENZ; 1986). 
Diante disso, são necessários estudos que avaliem os efeitos de drogas usadas de forma metafilática (ROMERO et al., 2013). Assim, a busca por novas alternativas terapêuticas é uma necessidade constante, principalmente para o controle da doença em sua forma subclínica, evitando manifestações clínicas e garantindo o adequado desenvolvimento e a máxima expressão produtiva dos animais. Recentemente, alguns compostos, entre eles o Toltrazuril 5\% (SARATSIS et al., 2013; VERONESI et al., 2011) têm sido indicados não apenas para o tratamento da coccidiose clínica em bezerras leiteiras, mas também como metafilaxia em casos subclínicos, na tentativa de eliminar o agente infeccioso, evitar a contaminação constante do ambiente e a infecção de um maior número de animais. Esta estratégia pode evitar o retardo no crescimento dos animais e também as perdas produtivas, as quais são comuns nestas situações (DAUSCHIES et al., 2007).

Assim, trabalhamos com a hipótese de que o Toltrazuril 5\% age interrompendo a excreção de oocistos, evitando a baixa eficiência alimentar e garantindo o adequado desenvolvimento dos animais. Diante disso, o objetivo deste estudo foi demonstrar a eficácia do Toltrazuril 5\% no tratamento da coccidiose subclínica, sobre a excreção de oocistos fecais e ganhos de peso de bezerras leiteiras naturalmente infectadas durante as primeiras semanas de vida.

\section{MATERIAL E MÉTODOS}

Este estudo foi realizado em uma fazenda leiteira localizada no município de Rio Grande (RS) $\left(32,8^{\circ} 16^{\prime} \mathrm{S}, 52,8^{\circ} 32^{\prime} \mathrm{L}\right)$, onde foram selecionadas 22 bezerras da raça Holandês com presença confirmada de oocistos nas fezes (no dia -7 do estudo). Os animais tinham entre 20 e 35 dias de idade e eram alojados em baias de $1 \mathrm{~m}^{2}$, de piso ripado, cobertas e suspensas a $1,5 \mathrm{~m}$ do solo, ou alocados em estacas individuais, diretamente no solo. Recebiam uma dieta composta por 5 litros de leite pasteurizado e alimento concentrado, ofertada duas vezes ao dia, e tinham livre acesso à água.

As bezerras não receberam nenhum tratamento com anticoccidianos antes da entrada no estudo, assim como não apresentaram diarreia no início do experimento. As mesmas foram divididas aleatoriamente nos seguintes grupos experimentais: Tratamento $(n=13)$, que receberam uma dose oral de $3 \mathrm{~mL} / 10 \mathrm{~kg}$ de peso vivo $\left(15 \mathrm{mg} / \mathrm{kg}\right.$ ) de Toltrazuril 5\% (Baycox ${ }^{\bullet}$, 
Bayer Health Care, São Paulo, Brasil) no dia 0, uma semana após a confirmação da eliminação de oocistos; e grupo Controle $(n=9)$, que receberam uma dose oral de $3 \mathrm{~mL} / 10 \mathrm{~kg}$ de peso vivo de solução de $\mathrm{NaCl}$ 0,9\%, como placebo.

Foram realizadas coletas de fezes diretamente da ampola retal nos dias $-7,0$ e 14 para a determinação da quantidade de ovos por grama de fezes (OOPG). A excreção de oocistos foi efetuada pela técnica de flutuação com solução de $\mathrm{NaCl}$ saturada, seguido por contagem em câmaras de McMaster (ECKERT et al., 1995).

O peso corporal dos animais foi avaliado nos dias 0, 14 e 32 do experimento, através de fita de pesagem (SUÑ́́ et al., 2011). O peso médio inicial das bezerras foi de 54,8 kg e 53,3 kg para os grupos Tratamento e Controle, respectivamente $(P \geq 0,05)$. $O$ desempenho de crescimento foi analisado calculando-se o ganho de peso entre o final do período do estudo (dia 32) e o início do tratamento (dia 0). O desenho experimental está ilustrado na Figura 1.

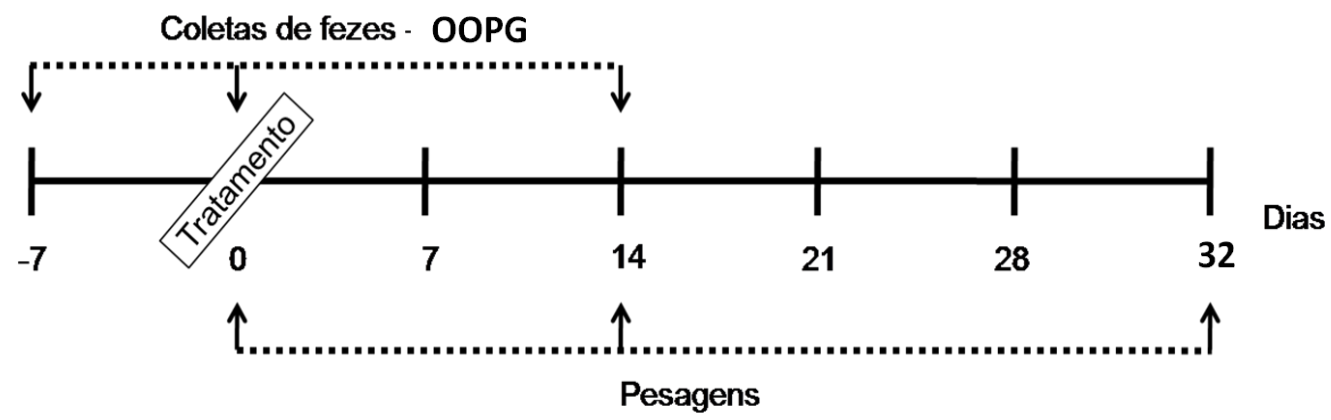

Figura 1 - Cronograma de coletas de fezes, exames de OOPG, aplicação de Toltrazuril 5\% e pesagem dos animais durante o período experimental.

\section{ANÁLISE ESTATÍSTICA}

Para a realização das análises estatísticas foi utilizado o programa estatístico SAS ${ }^{\oplus}$ Versão 9.0 (SAS Institute Inc., Cary, NC, EUA). Os resultados foram apresentados em média e erro padrão da média. Para os dados referentes ao peso corporal, quantidade de oocistos fecais e ganho médio diário (GMD) de peso entre os grupos, foi utilizada análise de variância ANOVA com medidas repetidas sendo as comparações de médias realizadas através do teste TukeyKramer no MIXED MODELS, com a utilização do modelo de comparação de grupos (controle 
$x$ tratamento). A comparação entre os grupos quanto ao percentual de animais eliminando oocistos foi apenas descrita. A diferença estatística foi definida como $P \leq 0,05$.

\section{RESULTADOS E DISCUSSÃO}

No manejo utilizado na propriedade, as bezerras permaneceram com as vacas no campo por pelo menos 24 horas após o parto, período que pode ser suficiente para a infecção com Eimeria spp. As avaliações coprológicas iniciaram a partir de 20 dias de idade (20 - 35 dias), pois a excreção de oocistos começa a partir de três semanas de idade após a ingestão de oocistos esporulados (CHARTIER; PARAUD, 2012).

Durante o experimento nenhum animal apresentou sinais clínicos de Eimeriose, configurando as infecções como subclínicas e condizentes com a quantidade de oocistos encontrados (Tabela 1). A classificação foi baseada em Mundt et al. (2005a), segundo o qual a eliminação de oocistos fecais é o parâmetro mais sensível para se avaliar a infecção por coccídeos.

Tabela 1 - Número médio (mínimo e máximo) de oocistos de Eimeria spp. em amostras fecais de bezerras leiteiras da raça Holandês antes e após o tratamento.

\begin{tabular}{|c|c|c|c|}
\hline \multirow{2}{*}{ Grupo } & \multicolumn{2}{|c|}{ Contagem média de oocistos (mínima e máxima) } & \multirow{2}{*}{ Valor de $\mathrm{P}$} \\
\hline & Dia 0 & Dia 14 & \\
\hline Tratamento $^{1}(n=13)$ & $926,7(0-5.600)^{\mathrm{a}}$ & $7(0-50)^{b}$ & 0,04 \\
\hline Controle $^{2}(n=9)$ & $242,5(0-1.300)^{a}$ & $523,7(0-3.900)^{\mathrm{a}}$ & 0,67 \\
\hline
\end{tabular}

De acordo com Mundt et al. (2005a), o principal objetivo do tratamento é diminuir ao máximo a excreção de oocistos. Neste caso, o Toltrazuril 5\% foi eficaz, já que os animais do grupo Tratamento apresentaram uma redução de 99,2\% na excreção de oocistos fecais 14 dias pós-tratamento (dia 14), enquanto que os animais do grupo Controle tiveram um aumento de 115,9\% (Tabela 1). Os resultados dos animais tratados estão em concordância 
com o trabalho realizado por Saratsis et al. (2013), em cordeiros, que encontraram mais de 90\% de redução na eliminação de oocistos quatro semanas após o tratamento, assim como trabalhos de Ghanem et al. (2008) e Mundt et al. (2005b) que encontraram resultados semelhantes.

Uma semana antes do início do experimento (dia -7), todas as bezerras estavam eliminando oocistos fecais. Após uma semana, observou-se uma flutuação no percentual de bezerras eliminando oocistos (redução de $39,7 \%$ e $65 \%$ para o grupo Tratamento e Controle, respectivamente). Estas variações são consideradas normais, em parte devido ao ciclo do parasita e ao fato de que estes animais podem ter desenvolvido imunidade contra as espécies de Eimeria spp. envolvidas (REEG et al., 2005). Além disso, consideramos que mesmo aqueles que não estavam eliminando oocistos no dia 0 , poderiam estar naturalmente infectados, pois apresentaram oocistos na semana anterior e não foram tratados.

Com relação às bezerras do grupo Tratamento eliminando oocistos no dia 0 , houve uma redução de $75 \%$ no número destes animais eliminando oocistos 14 dias após o tratamento, enquanto que não houve redução no grupo Controle. Estes resultados estão em concordância com os achados de Mundt et al. (2007) e demonstram a eficácia do Toltrazuril 5\% na diminuição do percentual de animais infectados, alertando para a importância do tratamento dos casos subclínicos, uma vez que não foi reduzido o percentual de infecção dos animais não tratados.

Apesar do caráter subclínico encontrado nas infecções, a diminuição do número de animais excretando oocistos e da quantidade de oocistos excretados é um importante achado, uma vez que essa forma subclínica também gera perdas econômicas nas bezerras, em virtude ao baixo ganho de peso decorrente da baixa conversão alimentar (FITZGERALD, 1980). Além disso, essa redução geral na eliminação de oocistos nos animais tratados com Toltrazuril 5\% também resulta em efeitos diretos na diminuição da pressão de infecção sobre as bezerras que serão posteriormente alojadas no mesmo local, resultando assim num controle da coccidiose por um maior período de tempo (MUNDT et al., 2005a). 
Apesar de que o objetivo deste trabalho não foi de comparar o Toltrazuril 5\% com nenhum outro produto disponível contra a coccidiose bovina, alguns trabalhos demonstram as vantagens deste produto frente a outras substâncias de mesma indicação, com algumas diferenças nas propriedades farmacocinéticas (maior meia vida plasmática) e espectro de ação frente a todos os estágios do ciclo da Eimeria (BALICKA-RAMISZ, 1999; HABERKORN; STOLTEFUSS, 1987).

Os efeitos do fármaco sobre o ganho de peso e ganho médio diário em quilos (GMD) dos grupos estão apresentados na Tabela 2.

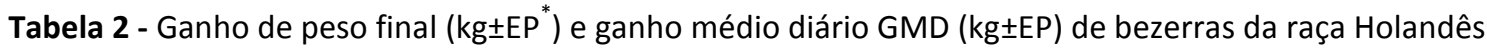
naturalmente infectadas por Eimeria spp., tratadas com Toltrazuril 5\%.

\begin{tabular}{|c|c|c|c|}
\hline \multirow{2}{*}{$\begin{array}{l}\text { Período (dias) } \\
\qquad 0-32\end{array}$} & \multicolumn{2}{|c|}{ Grupos } & \multirow{2}{*}{ Valor de P } \\
\hline & Tratamento $^{1}(n=13)$ & Controle $^{2}(n=9)$ & \\
\hline Peso (kg士EP) & $10,7 \pm 1,4^{a}$ & $7,3 \pm 1,8^{a}$ & 0,15 \\
\hline GMD (kg士EP) & $0,334 \pm 0,04^{a}$ & $0,229 \pm 1,80^{a}$ & 0,15 \\
\hline
\end{tabular}

\footnotetext{
${ }^{1} 3 \mathrm{~mL} / 10 \mathrm{~kg}$ de peso vivo (15 mg/kg) de Toltrazuril 5\% (Baycox ) no dia 0;

${ }^{2} 3 \mathrm{~mL} / 10 \mathrm{~kg}$ de peso vivo de solução de $\mathrm{NaCl}$ 0,9\%, como placebo, no dia 0 .

*Erro padrão da média.
}

Letras iguais na linha não diferem estatisticamente $(P>0,05)$.

Os efeitos deletérios da infecção subclínica são eliminados quando o ciclo da Eimeria spp. é interrompido, resultando num maior ganho de peso (DAUGSCHIES et al., 2007). Entretanto, no presente estudo não foram encontradas diferenças entre o ganho de peso e o ganho médio diário entre os grupos (Tabela 2 ) possivelmente pelo número limitado de animais avaliados, perfil subclínico da infecção e pelo curto período de estudo. Contudo, a diferença observada de 3,4 kg de peso vivo em 32 dias deve ser considerada, justamente pelos fatores acima comentados.

A diferença no ganho de peso diário de $105 \mathrm{~g} /$ dia a mais para os animais que receberam o Toltrazuril 5\% é semelhante ao encontrado por Lassen e Ostergaard (2012), portanto, apesar da ausência de diferença estatística, é um importante resultado considerando a forma subclínica da infecção e o curto período de tempo do estudo. 
Ainda, ao final do período de avaliação (32 dias após o tratamento) as bezerras do grupo Tratamento apresentaram $4,9 \mathrm{~kg}$ a mais de peso $(P \leq 0,05)$ em relação as do grupo Controle $(65,5 \pm 2,1 \mathrm{~kg}$ e $60,6 \pm 2,0 \mathrm{~kg}$ ) (Figura 2), demonstrando assim os efeitos prolongados do Toltrazuril 5\% sobre o desenvolvimento das bezerras. Os achados estão em concordância com Mundt et al. (2005b) e Veronesi et al. (2011) e são uma importante resposta da ação do Toltrazuril 5\% em evitar um aumento na infecção naqueles animais já infectados. Daugschies et al. (2007) observaram depressão no ganho de peso nos animais acometidos pela coccidiose subclínica e que não receberam nenhum tratamento, suportando assim as observações de Fitzgerald (1980), de que as infecções leves podem afetar o ganho de peso.

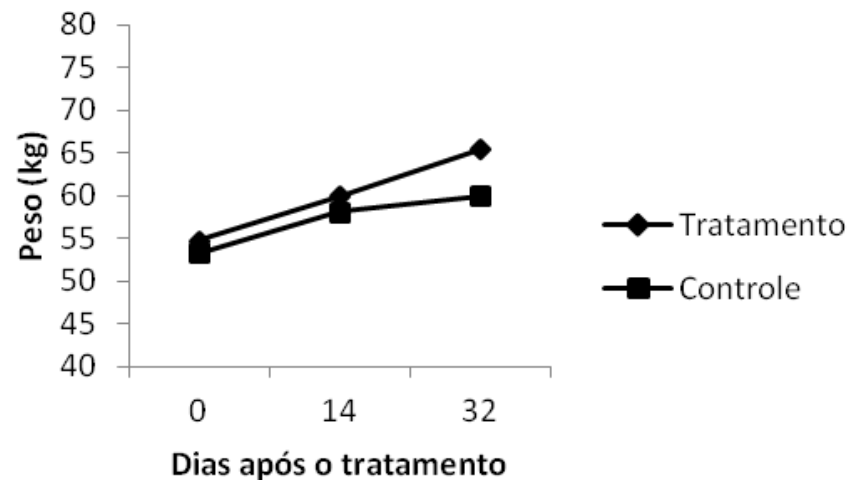

Figura 2 - Peso corporal de bezerras naturalmente infectadas com Eimeria spp. e submetidas a tratamento com Toltrazuril 5\%.

Tratamento ( $\mathrm{n}=13): 3 \mathrm{~mL} / 10 \mathrm{~kg}$ de peso vivo (15 mg/kg) de Toltrazuril 5\% (Baycox ) no dia 0.

Controle ( $n=9): 3 \mathrm{~mL} / 10 \mathrm{~kg}$ de peso vivo de solução de $\mathrm{NaCl}$ 0,9\%, como placebo, no dia 0 .

$* \mathrm{P} \geq 0,05 ; * * \mathrm{P} \leq 0,05$.

Muitos trabalhos demonstram os resultados no ganho de peso em animais severamente infectados, ou seja, com grande eliminação de oocistos e possivelmente até o desenvolvimento da forma clínica da doença (GHANEM et al., 2008; MUNDT et al., 2005b). Entretanto, as maiorias das infecções a campo são contínuas e com baixas doses de oocistos, provenientes de um ambiente naturalmente contaminado, o que é mais provável de refletir as condições naturais das bezerras e o impacto posterior na taxa de crescimento (DAUGSCHIES; NAJDROWSKI, 2005). Daugschies et al. (2007) observaram que, apesar dos animais acometidos clinicamente pela coccidiose apresentarem maiores perdas de peso no 
período de 3 semanas, a maior prevalência de casos subclínicos faz com que, no total, os casos subclínicos superem os clínicos na perda de peso, ou seja, mesmo pequenas diferenças de ganhos médios diários perfazem um montante considerável no curto/médio prazo.

De acordo com os achados do presente estudo, bezerras naturalmente expostas à infecção por Eimeria spp. e submetidas à metafilaxia com Toltrazuril 5\%, apresentaram redução na eliminação de oocistos fecais e um maior peso ao final do período experimental. A razão para utilizar um tratamento metafilático em um sistema de criação baseia-se em fatores específicos de cada propriedade e na relação custo/benefício. Os custos do tratamento anticoccidiano precisam considerar o valor econômico do adicional ganho de peso e o risco da ocorrência de surtos clínicos de coccidiose na propriedade.

\section{CONCLUSÃO}

O tratamento com o Toltrazuril 5\% foi eficaz na redução da eliminação de oocistos, na diminuição do percentual de animais infectados por coccidiose subclínica e no peso corporal ao final do período experimental, confirmando seus benefícios produtivos. Isso torna o Toltrazuril 5\% uma alternativa viável para minimizar os efeitos deletérios da coccidiose subclínica e evitar o aparecimento da forma clínica da doença, evitando assim o retardo no desenvolvimento durante a fase inicial de criação. 


\title{
EFFECT OF A TOLTRAZURIL 5\% TREATMENT ON OOCYST EXCRETION AND WEIGHT GAIN IN DAIRY CALVES WITH SUBCLINICAL COCCIDIOSIS
}

\begin{abstract}
The aim of this study was to demonstrate the effectiveness of Toltrazuril $5 \%$ in the treatment of subclinical coccidiosis by evaluating the excretion of fecal oocysts and weight gain of dairy calves naturally infected with Eimeria spp. during the first three weeks of life. Twenty-two Holstein calves with confirmed presence of oocysts in the feces (day -7 of the study) were selected. The animals were between 20 and 35 days of age and received the same diet. Calves were divided into the following groups: Treatment $(n=13)$ who received an oral dose of $3 \mathrm{~mL} / 10 \mathrm{~kg}$ live weight of Toltrazuril $5 \%$ on day 0 , a week after confirmation of elimination (day -7$)$ and Control group $(n=9)$ who received an oral dose of 3 $\mathrm{mL} / 10 \mathrm{~kg}$ live weight of $0.9 \% \mathrm{NaCl}$ solution as placebo. The collection of fecal samples for evaluation of oocysts shedding was performed on days $-7,0$, and 14 , while the body weight evaluation was performed on days 0,14 and 32. Treatment with Toltrazuril 5\% decreased the oocysts shedding and the percentage of infected calves and have provided a greater weight gain to the animals which received the treatment. Thus, the Toltrazuril $5 \%$ may be a viable alternative to minimize the deleterious effects of subclinical coccidiosis and to prevent the onset of clinical signs in dairy calves, thus avoiding the a delay in the development during the initial phase of life.
\end{abstract}

Keywords: Coccidiosis. Toltrazuril. Dairy calves. Oocysts. DWG. 


\section{INFLUENCIA DEL TOLTAZURIL 5\% EN OOQUISTES EXCRETADOS Y GANANCIA DE PESO EM BECERRAS CON COCCIDIOSE SUBCLÍNICA}

\section{RESUMEN}

$\mathrm{E}$ I objetivo de este estudio fue demostrar la eficacia de Toltrazuril 5\% en el tratamiento de la coccidiosis subclínica mediante la evaluación de la excreción fecal de ooquistes y de la ganancia de peso de las terneras lecheras infectadas de forma natural con Eimeria spp. durante las primeras semanas de vida. Fueron seleccionadas 22 terneras Holando con presencia confirmada de ooquistes en las heces (día -7 del estudio). Los animales tenían entre 20 y 35 días de edad y recibieron la misma dieta. Las terneras se dividieron en los siguientes grupos: tratamiento $(n=13)$ que recibieron una dosis oral de 3 $\mathrm{mL} / 10 \mathrm{~kg}$ peso vivo de Toltrazuril $5 \%$ en el día 0 , una semana después de la confirmación de la eliminación de ooquistes (día -7) y el grupo control $(n=9)$ que recibieron una dosis oral de $3 \mathrm{~mL} / 10 \mathrm{~kg}$ peso vivo de solución de $\mathrm{NaCl}$ al 0,9\% como el placebo. La obtención de muestras de heces para la evaluación de la eliminación de ooquistes se realizó en los días -7, 0 y 14, mientras que la evaluación del peso corporal se realizó en los días 0, 14 y 32. El tratamiento con Toltrazuril 5\% disminuyó la excreción ooquistes y el porcentaje de terneras infectadas, y además determinó un mayor peso vivo final para las que recibieron el tratamiento. Por lo tanto, la Toltrazuril 5\% se presenta como una alternativa viable para minimizar los efectos deletéreos de la coccidiosis subclínica y prevenir la aparición de los signos clínicos, evitando de este modo la demora en el desarrollo durante la fase inicial de la crianza.

Palabras clave: Coccidiosis. Toltrazuril. Terneras lecheras. Ooquistes. GMD.

\section{REFERÊNCIAS}

BALICKA-RAMISZ, A. The usefulness of Baycox (Bayer) for coccidiosis control of lambs. Wiad Parazytology, v. 45, p. 187-191, 1999.

BANGOURA, B.; DAUGSCHIES, A. Parasitological and clinical parameters of experimental Eimeria zuernii infection in calves and influence on weight gain and haemogram.

Parasitology Research, v. 100, p. 1331-1340, 2007.

CHARTIER, C.; PARAUD, C. Coccidiosis due to Eimeria in sheep and goats, a review. Small Ruminant Research, v. 103, p. 84-92, 2012.

DAUGSCHIES, A.; NAJDROWSKI, M. Eimeriosis in cattle: current understanding. Journal of Veterinary Medicine, v. 52, p. 417-427, 2005. 
DAUGSCHIES, A.; AGNEESSENS, J.; GOOSSENS, L.; et al. The effect of a metaphylatic treatment with diclazuril (Vecoxan ) on the oocyst excretion and growth performance of calves exposed to a natural Eimeria infection. Veterinary Parasitology, v. 149, p. 199-206, 2007.

ECKERT, J.; BRAUN, R.; SHIRLEY, M. W.; et al. Guidelines on techniques in coccidiosis research. Office for official publications of the European communities, Luxemburg, 1995.

ERNST J. V.; BENZ, G. W. Intestinal coccidiosis in cattle. In: ERNST J. V.; BENZ, G. W. The veterinary clinics of North America/parasites:epidemiology and control. W.B. Philadelphia, PA: Saunders Company, 1986.

FITZGERALD, P. R. The economic impact of coccidiosis in domestic animals. Advances in Veterinary Sciences and Comparative Medicine, v. 24, p. 121-143, 1980.

GHANEM, M. M.; RADWAAN, M. C.; MOUSTAFA, A. M. M.; et al. Comparative therapeutic effect of toltrazuril, sulphadimine and amprolium on Eimeria bovis and Eimeria zuernii given at different times following infection in buffalo calves (Bubalus bubalis). Preventive Veterinary Medicine, v. 84, p. 161-170, 2008.

HABERKORN, A.; STOLTEFUSS, J. Studies on the activity spectrum of Toltrazuril: a new anticoccidial agent. Veterinary Medicine Nachr., v. 1, p. 22-32, 1987.

HOOSHMAND-RAD, P.; SVENSSON, C.; UGGLA, A. Experimental Eimeria alabamensis infection in calves. Veterinary Parasitology, v. 53, p. 23-32, 1994.

LASSEN, B.; OSTERGAARD, S. Estimation of the economical effects of Eimeria infection in Estonian dairy herds using a stochstic model. Preventive Veterinary Medicine, v. 106, p. 258-265, 2012.

LIMA, J. D. Coccidiose dos ruminantes domésticos. Revista Brasileira de Parasitologia Veterinária, v. 13, suplemento 1, p. 9-11, 2004.

MATJILA, P. T.; PENZHORN, B. L. Occurrence and diversity of bovine coccidia at three localities in South Africa. Journal of Veterinary Parasitology, v. 104, p. 93-102, 2002.

MUIRHEAD, S. Coccidiosis infections often go undetected in beef, dairy cattle. Feedstuffs, v. 15, p. 87, 1989.

MUNDT, H. C.; BANGOURA, B.; MENGEL, H.; et al. Control of clinical coccidiosis of calves due to Eimeria bovis and Eimeria zuernii with Toltrazuril under field conditions. Parasitology Research, v. 97, p. 134-142, 2005a. 
MUNDT, H. C.; BANGOURA, B.; RINKE, M.; et al. Pathology and treatment of Eimeria zuernii coccidiosis in calves: Investigation in a infection model. Parasitology International, v. 54, p. 223-230, 2005b.

MUNDT, H. C.; RÖDDER, F.; MENGEL, H.; et al. Control of coccidiosis due to Eimeria bovis and Eimeria zuernii in calves with Toltrazuril under field conditions in comparison with Diclazuril and untreated controls. Parasitology Research, v. 101, Supplement 1, p. 94-101, 2007.

REEG, K. J.; GAULY, M.; BAUER, C.; et al. Coccidial infections in housed lambs: oocyst excretion, antibody levels and genetic influences on the infection. Veterinary Parasitology, v. 127, p. 209-219, 2005.

ROMERO, J.; SANABRIA, R.; TRAVERÍA, G.; et al. Short Communication - Metaphylactic effect of Diclazuril $0.25 \%$ in suckling beef calves, during a coccidiosis outbreak in extensive farming. Veterinary Parasitology, v. 193, p. 277-280, 2013.

SARATSIS, A.; KARAGIANNIS, I.; BROZOS, C.; et al. Lamb eimeriosis: applied treatment protocols in dairy sheep production systems. Veterinary Parasitology, v. 196, p. 56-63, 2013.

SPADETTO, R. M., TAVELA, A. O. Importância do manejo dos neonatos para um aumento do número de bezerros desmamados. Revista Científica Eletrônica de Medicina Veterinária, ano XI, n²1, Jul. 2013.

SUÑÉ, R. W. M. S.; MÜLLER, M.; D’AVILA, P. P.; et al. Correlação entre fita de pesagem corporal e balança na determinação do peso de bezerras das raças Holandesa e Jersey. XX Congresso de Iniciação Científica da Universidade Federal de Pelotas, Pelotas (RS), 2011.

VERONESI, F.; DIAFERIA, M.; VIOLA, O.; et al. Short Communication - Long-term effect of toltrazuril on growth performances of dairy heifers and beef calves exposed to natural Eimeria zuernii and Eimeria bovis infections. The Veterinary Journal, v. 190, p. 296-299, 2011. 